\title{
Thermal inspection of composite honeycomb structures
}

\author{
Joseph N. Zalameda* and F. Raymond Parker \\ NASA Langley Research Center Hampton, VA 23681-2199; \\ VA 23681-2199
}

\begin{abstract}
Composite honeycomb structures continue to be widely used in aerospace applications due to their low weight and high strength advantages. Developing nondestructive evaluation (NDE) inspection methods are essential for their safe performance. Pulsed thermography is a commonly used technique for composite honeycomb structure inspections due to its large area and rapid inspection capability. Pulsed thermography is shown to be sensitive for detection of face sheet impact damage and face sheet to core disbond. Data processing techniques, using principal component analysis to improve the defect contrast, are presented. In addition, limitations to the thermal detection of the core are investigated. Other NDE techniques, such as computed tomography X-ray and ultrasound, are used for comparison to the thermography results.
\end{abstract}

Keywords: Thermal nondestructive evaluation, face sheet to core disbond, face sheet delamination, honeycomb composite structure inspection

\section{INTRODUCTION}

Composite honeycomb structures continue to be used widely in aerospace applications due to low weight and high strength requirements. There is a growing interest in the application of thermal methods for nondestructive evaluation (NDE) of composite sandwich structures [1-3]. Some of the advantages of thermal NDE are noncontact, rapid, capable of imaging large areas, applicable to complex geometries, and quantitative. The technique is safe where only a small amount of heat is applied to the surface of the structure. Thermography has shown good potential for detection of various defects in composite structures. In composite structures, defects such as delaminations, disbonds, gross porosity, and fiber volume fraction variations are detectable using thermography.

Specific to composite honeycomb structures, face sheet delaminations, face sheet to core disbonds, and core crushing are defects of interest. Typically the composite face sheets are relatively thin compared to the overall thickness and therefore, thermography is effective in detection of face sheet delaminations. Detection of the face sheet to core disbonds is critical. Under load, face sheet to core disbonds can grow leading to disbond buckling failure and this can lead to catastrophic structural failure [4]. Thermography has the potential to detect face sheet to core disbonds. In this work, the detection limitations of face sheet to core disbonds are investigated using analytical and finite element thermal modeling. Flash thermography results are presented on composite honeycomb samples with face sheet delaminations, face sheet to core disbonds, and core crushing. The modeling results are also used to determine how to differentiate between the face sheet delaminations and face sheet bonding.

A 1-dimensional (1-D) analytic model and 2-dimensional (2-D) finite element model were used. The 1-D analytical model was used to provide an understanding of the effect of the adhesive between the face sheet and core. The 2-D finite element model was used for two purposes: first to determine the optimal thermal processing parameters for detection of face sheet delamination damage and face sheet to core disbonds and second to determine the thermal detection limitations caused by the face sheet to core thickness ratio. These models were used for the study of thermography as a technique for detection of damage and differentiating between impact damage and face sheet disbonds using early time and late time analysis. Infrared flash thermography was acquired on composite specimens with differing damage types. Data processing techniques using principal component analysis (PCA) to improve the defect contrast were used to process the thermal data. Lastly, other NDE techniques such as computed tomography (CT) $\mathrm{X}$-ray and ultrasound are compared to the thermography results.

*joseph.n.zalameda@nasa.gov; phone 1 757-864-4793; fax 1 757-864-4914; http://nde.larc.nasa.gov/majorcap.shtml 


\section{SAMPLE CONFIGURATION}

The configuration of the composite sandwich samples studied is shown in figure 1. The sandwich structure was comprised of an aluminum hexagonal core with outer graphite plain weave composite face sheets. The core thickness was $2.54 \mathrm{~cm}$ and the composite face sheet thickness was approximately $0.1 \mathrm{~cm}$. The core cell wall thickness was approximately $0.02 \mathrm{~cm}$ with a cell size of $0.32 \mathrm{~cm}$. The ratio of the face sheet to core wall thickness is 5 to 1 .

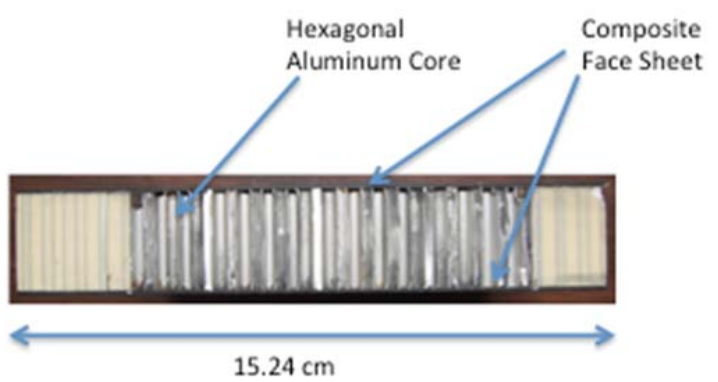

Figure 1. Typical configuration of composite face sheet with aluminum core.

\section{MODELING RESULTS}

The modeling efforts included a 1-D approach to investigate the effects of the adhesive between the face sheet and core. A multi-layer 1-D thermal model was used by comparing the difference in the thermal response between the face sheet bonded directly to the core and a thin layer of adhesive between the face sheet and core. From these results, a 2-D finite element model was configured similar to the right image of figure 2 using a " $T$ " configuration. This was used to study the effect of the lateral heat flow in reducing thermal contrast for imaging a core disbond. Simulated camera noise was added to the model results and was determined from actual thermal data. An X-ray CT image showing the aluminum core bonded to a composite face sheet is shown in the left image of figure 2. For both of these models, the fillet bonds between the core and adhesive along the length of the core wall were not considered.

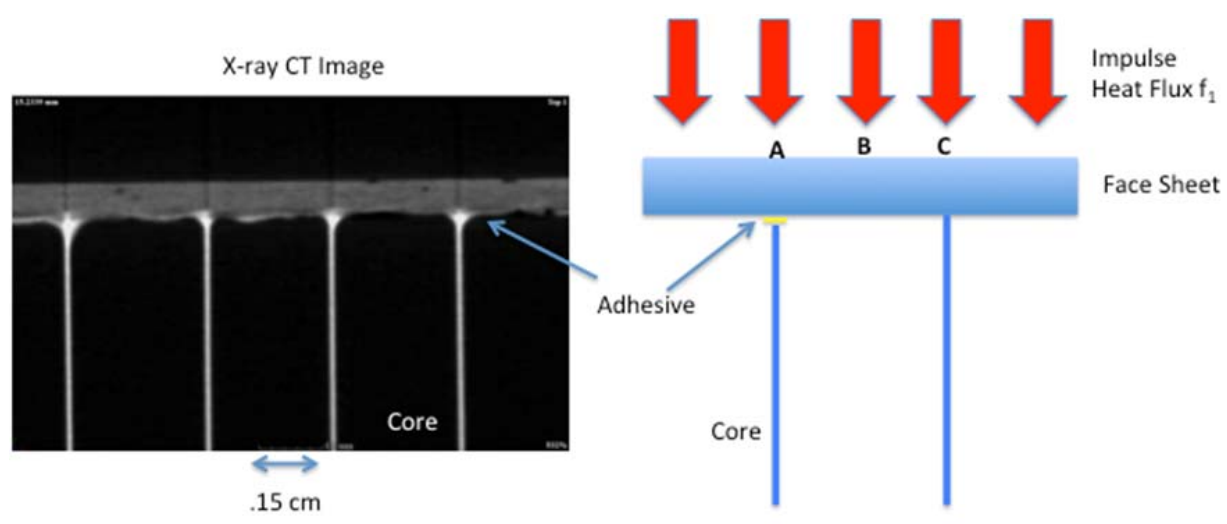

Figure 2. X-ray CT image (left) showing actual thermal configuration of attached core to the face sheet and thermal model configuration (right).

\subsection{1-D Modeling}

The 1-D analytical model provided an understanding of the effect of the adhesive between the face sheet and core is given in equation (1) [5]. The multilayered model includes the face sheet composite layer, the adhesive layer and the core layer. A contrast function is defined by the temperature difference response to impulse flux heating at the surface between points $\mathrm{A}, \mathrm{B}$, and $\mathrm{C}$ as shown in figure 1. For point A, the 1-D analytical model contains 3 layers: the face sheet, 
the adhesive, and the core, equation (1). For points B and C the analytical model contains 1 layer (face sheet layer only) and 2 layers (face sheet and the core), equations (2) and (3) respectively. The equations are given below as:

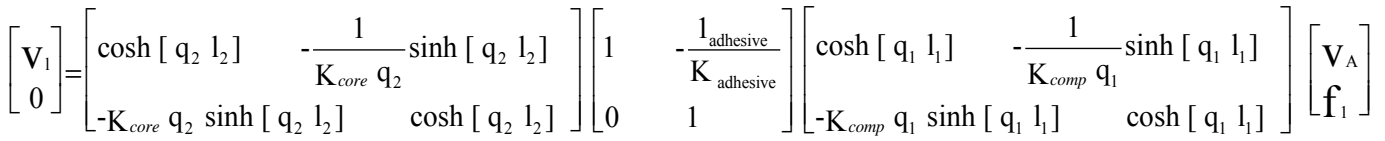

$$
\begin{aligned}
& {\left[\begin{array}{c}
\mathrm{V}_{2} \\
0
\end{array}\right]=\left[\begin{array}{ccc}
\cosh \left[\mathrm{q}_{1} \mathrm{l}_{1}\right] & -\frac{1}{\mathrm{~K}_{\operatorname{comp}} \mathrm{q}_{1}} \sinh \left[\mathrm{q}_{1} \mathrm{l}_{1}\right] \\
-\mathrm{K}_{\text {comp }} \mathrm{q}_{1} \sinh \left[\mathrm{q}_{1} \mathrm{l}_{1}\right] & \cosh \left[\mathrm{q}_{1} \mathrm{l}_{1}\right]
\end{array}\right]\left[\begin{array}{c}
\mathrm{V}_{\mathrm{B}} \\
\mathrm{f}_{1}
\end{array}\right]}
\end{aligned}
$$

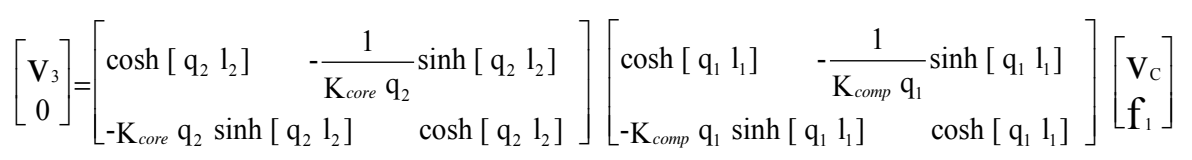

$$
\begin{aligned}
& \text { where } \mathrm{q}_{1}=\sqrt{\frac{\mathrm{s}}{\alpha_{\text {comp }}}} \text { and } \mathrm{q}_{2}=\sqrt{\frac{\mathrm{s}}{\alpha_{\text {core }}}} \text {. }
\end{aligned}
$$

The model can be solved analytically in the Laplace domain [6] where $\mathrm{V}_{\mathrm{A}}, \mathrm{V}_{\mathrm{B}}$ and $\mathrm{V}_{\mathrm{C}}$ are the 3 layer, 1 layer, and 2 layer surface temperature responses in the Laplace domain respectively, $\mathrm{f}_{1}$ is the instantaneous heat flux input estimated to be approximately $1 \mathrm{cal} / \mathrm{cm}^{2}$ [7], $l_{1}$ is the face sheet composite layer thickness $=0.1 \mathrm{~cm}, l_{2}$ is the core layer thickness $=2.54$ $\mathrm{cm}, \mathrm{K}_{\text {comp }}$ is the composite thermal conductivity $=0.0021 \mathrm{cal} / \mathrm{cm} \mathrm{sec}{ }^{\circ} \mathrm{C}, \mathrm{K}_{\text {aluminum }}$ is the aluminum thermal conductivity $=0.33 \mathrm{cal} / \mathrm{cm} \mathrm{sec}{ }^{\circ} \mathrm{C}, \alpha_{\text {core }}$ is the composite thermal diffusivity $=0.004 \mathrm{~cm}^{2} / \mathrm{sec}, \alpha_{\text {aluminum }}$ is the aluminum thermal diffusivity $=0.53 \mathrm{~cm}^{2} / \mathrm{sec}, \mathrm{K}_{\text {adhesive }}$ is the adhesive thermal conductivity $=0.0006 \mathrm{cal} / \mathrm{cm} \mathrm{sec}{ }^{\circ} \mathrm{C}, 1_{\text {adhesive }}$ is the estimated adhesive thickness $=0.001 \mathrm{~cm}[8]$, and $\mathrm{s}$ is the Laplace complex argument. The temperature results are normalized before taking the difference. The results are shown in figure 3 where the contrast between the model with adhesive and no adhesive $\left(\mathrm{V}_{\mathrm{A}}-\mathrm{V}_{\mathrm{C}}\right)$ is plotted. In addition, the difference between the face sheet only and face sheet and core $\left(\mathrm{V}_{\mathrm{B}}-\right.$ $\mathrm{V}_{\mathrm{C}}$ ) is plotted and this provides the core contrast. As compared to the core contrast the adhesive difference response is minimal.

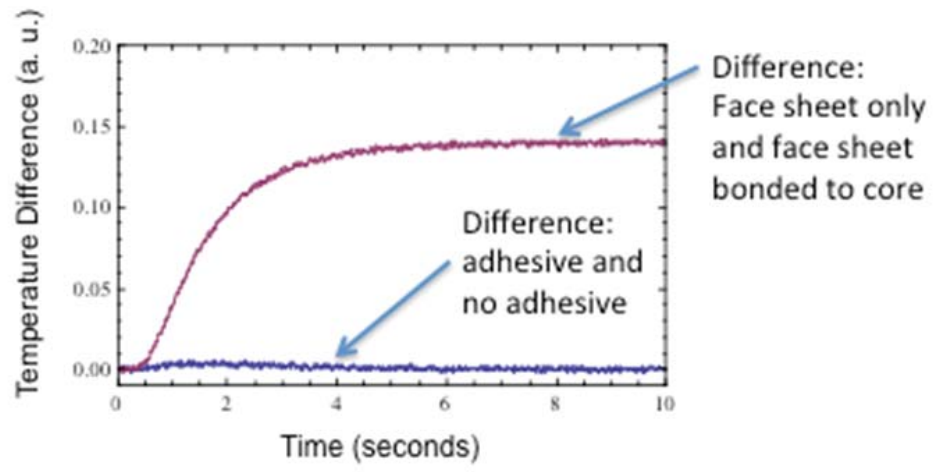

Figure 3. 1-D model temperature difference of adhesive and no adhesive compared to face sheet and face sheet and core.

\subsection{2-D Finite Element Modeling}

The 2-D finite element method (FEM), via a commercially available software package [9], was used to determine the thermal detection limitations caused by the face sheet to core thickness ratio. The face sheet thickness to core thickness 
ratios studied were $4: 1,5: 1,6: 1,7: 1$ and $8: 1$. These models were simplified using the face sheet bonded directly to the core (no adhesive) based on the 1-D results. An example finite element analysis model of a composite face sheet with aluminum core is shown in figure 4. The 2-D model contains 1038 elements and 620 nodes. The face sheet thickness is $0.1 \mathrm{~cm}$ and the aluminum core cell wall thickness is $0.020 \mathrm{~cm}$. The ratio of the face sheet to core thickness is $5: 1$. The width of the face sheet is $0.5 \mathrm{~cm}$ and the length of the core is $0.4 \mathrm{~cm}$. The surface temperature as a function of time is also shown in figure 4 . For early times around 0.3 seconds there is no contrast caused by the core. This reveals that defects within the face sheet can be detected around this time and therefore be differentiated from core defects. Later in time, around 0.97 seconds, the aluminum core lowers the temperature at the surface over the core. The temperature contrast fades away around 8.3 seconds. Figure 5 shows a comparison between the 2-D FEM and the 1-D analytical model results. For early times up to around 0.75 seconds, there is good agreement between the 2 models. Past 0.75 seconds the models diverge and as expected the 2-D model would predict lower temperature contrast since the lateral heat flow is taken into account. Comparisons to 2-D model results, with camera noise added, of the contrast evolution vs. time for various face sheet to core cell wall thickness ratios are shown in the right plot of figure 5. The temperature difference was calculated by subtracting a point over the core from a point away from the core. The core wall thickness is $0.020 \mathrm{~cm}$ and the thickness of the face sheet values are $0.08,0.10,0.12,0.14$, and $0.16 \mathrm{~cm}$, resulting in face sheet thickness to core thickness ratios of 4:1, 5:1, 6:1, 7:1 and 8:1 respectively. The temperature difference is barely above the noise for the 8:1 ratio configuration indicating a face sheet to core disbond would not be detectable.
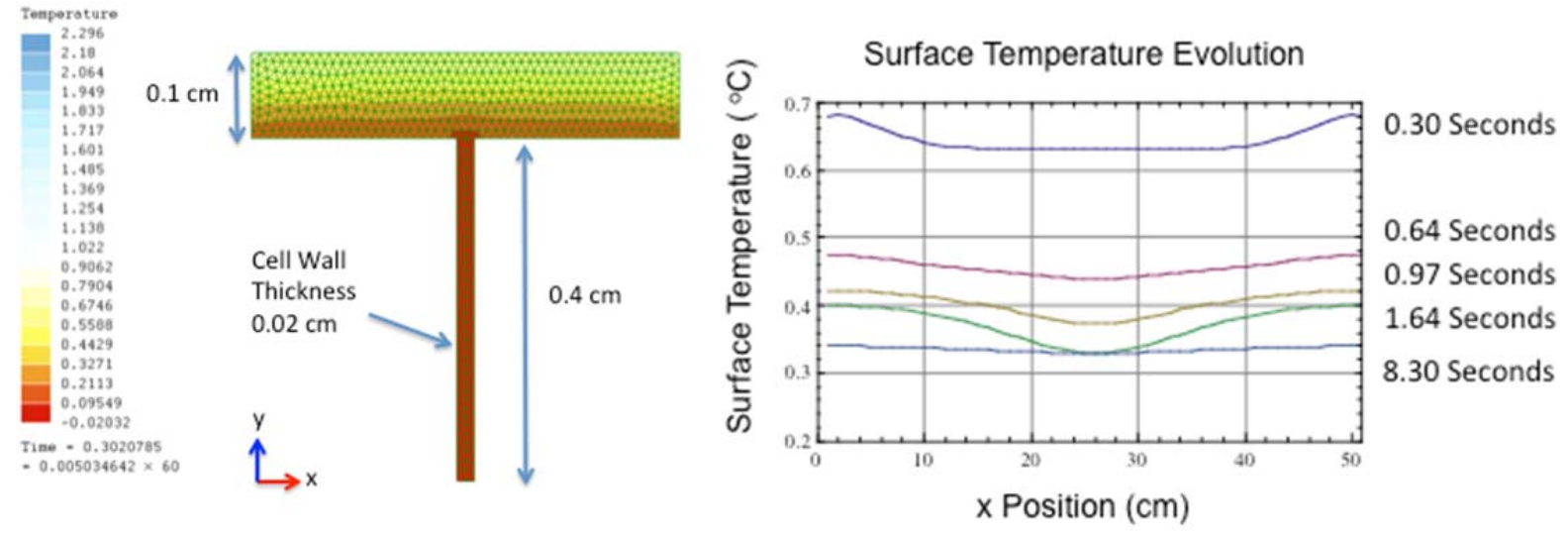

Figure 4. Finite element model of a composite face sheet with aluminum core (left image) and corresponding surface temperature evolution (right plot).
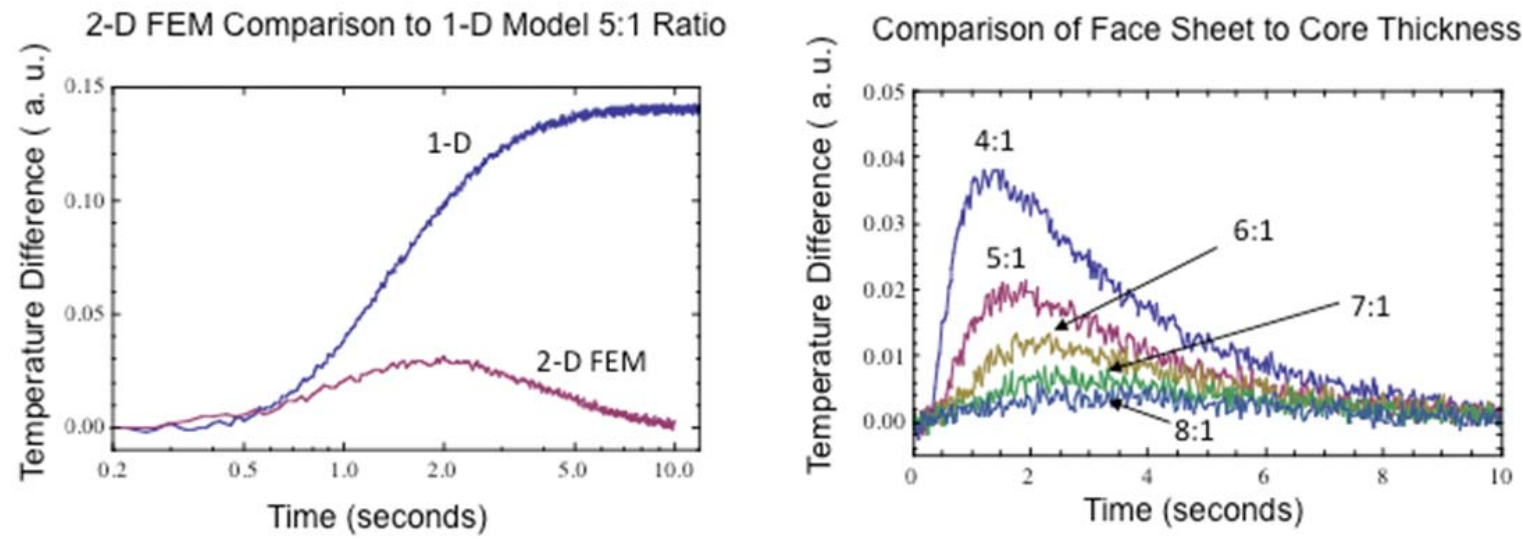

Figure 5. Contrast evolution comparison of 2-D finite element model comparison to 1-D analytical model (left plot) and temperature difference comparison of face sheet to core cell wall thickness ratios (right plot).

\section{DATA PROCESSING AND RESULTS}

Thermographic single side flash inspection data was acquired using a commercially available thermal inspection system. The thermal inspection system uses two flash lamps that are mounted within a hood to contain the flash. The flash power 
is provided from two power supplies delivering $4.8 \mathrm{~kJ}$ of energy to the flash tubes. The flash duration is typically less than 10 milliseconds and is instantaneous compared to the frame rate of the camera and the thermal response time of the composite [10]. An infrared camera is used to record the surface temperature temperature response. The camera operates in the mid infrared band with a pixel resolution of $640 \times 512$. The camera sensitivity or noise equivalent temperature difference, quoted by the manufacturer, is less than 0.02 Kelvin. The camera was configured with a $25 \mathrm{~mm}$ germanium optical lens. The data was acquired at $60 \mathrm{~Hz}$ for 720 frames. The total acquisition time was 12 seconds.

\subsection{Data Processing}

Principal component analysis (PCA) is common for processing of thermal data [11,12]. This algorithm is based on decomposition of the thermal data into its principal components or eigenvectors. Singular value decomposition is a routine used to find the singular values and corresponding eigenvectors of a matrix. Since thermal NDE signals are slowly decaying waveforms, the predominant variations of the entire data set are usually contained in the first or second eigenvectors, and thus account for most of the data variance of interest. The PCA is computed by defining a data matrix A, where the time variations are along the columns and the spatial image pixel points are row-wise. The matrix $\mathrm{A}$ is adjusted by subtracting the mean along the time dimension. The matrix A can then be decomposed using singular value decomposition as:

$$
A^{\mathrm{T}} A=\mathrm{U}^{*} \Gamma^{2} * \mathrm{U}^{\mathrm{T}}
$$

where $\Gamma$ is a diagonal matrix containing the squares of the singular values and $U$ is an orthogonal matrix, which contains the basis functions or eigenvectors describing the time variations. The eigenvectors can be obtained from the columns of U. The PCA image is calculated by dot product multiplication of the selected eigenvector times the temperature response (data matrix A), pixel by pixel. Typically the first or second eigenvector PCA image provides good contrast for defect detection. The first eigenvector is dominated by the transient cool down of the face sheet layer and is more suited for early time detection of face sheet delamination. The second eigenvector is less dominant and defines the temporal contrast evolution of the face sheet bonded to the core. This is shown in figure 6 where the $2^{\text {nd }}$ eigenvectors, determined from the 2-D model data for each of the thickness to core ratios, are plotted for comparison (no camera noise added). The maximum contrast point moves later in time due to the increases in the face sheet thickness, as expected. This indicates the $2^{\text {nd }}$ eigenvector is the best candidate for imaging the face sheet to core disbond regions. Also the maximum contrast points determined from the $2^{\text {nd }}$ eigenvectors are different compared to the maximum points determined from the difference calculation with no camera noise added (see table 1 in figure 6). While both agree with increasing face sheet thickness the maximum point moves later in time; the maximum contrast time determined from the $2^{\text {nd }}$ eigenvector is more accurate because all the surface points are used from the model instead of only the 2 surface points used in the difference calculation. Also shown in figure 6, the 8:1 ratio eigenvector curve does not have a clear maximum point, thus indicating the core response is not as dominant in the data and cannot be detected as confirmed by the 8:1 ratio temperature difference plot in figure 5 (especially with camera noise added).

Comparison of $2^{\text {nd }}$ Eigenvectors

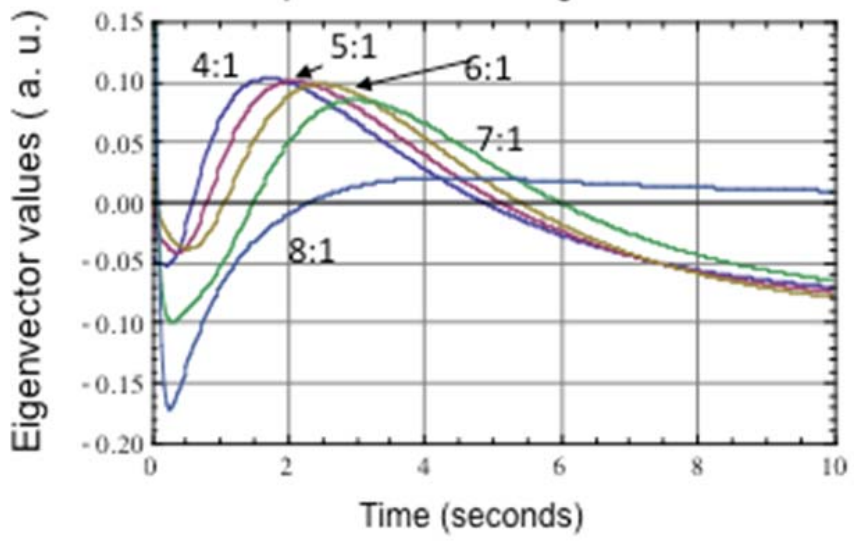

Table 1. Comparison of maximum contrast times.

\begin{tabular}{|l|l|l|}
\hline $\begin{array}{l}\text { Thickness to } \\
\text { Core Ratio }\end{array}$ & $\begin{array}{l}\text { Temperature } \\
\text { Difference Max } \\
\text { Time (seconds) }\end{array}$ & $\begin{array}{l}2^{\text {rd }} \text { Eigentector } \\
\text { Local Max Time } \\
\text { (seconds) }\end{array}$ \\
\hline $4: 1$ & 1.31 & 1.84 \\
\hline $5: 1$ & 1.70 & 2.07 \\
\hline $6: 1$ & 2.17 & 2.47 \\
\hline $7: 1$ & 2.61 & 2.98 \\
\hline $8: 1$ & 3.04 & 4.35 \\
\hline
\end{tabular}

Figure 6. $2^{\text {nd }}$ Eigenvectors determined from the 2-D FEM results (left plot) and table of maximum contrast times determined from temperature difference calculations (from figure 5) and the local maximum contrast time from $2^{\text {nd }}$ eigenvector calculations. 


\subsection{Processing Results and Comparison to Computed Tomography X-ray and Ultrasonics}

Based on the 2-D modeling results from sections 3.2 and 4.1, an early time window can be used for detection of defects within the composite face sheet. A late time window can be used to detect a face sheet to core disbond. Shown in figure 7 is an early time $(0.33-1.5$ seconds) PCA image of a composite honeycomb sample with impact damage defects (7 total impacts). The impact damage shows up as lighter regions. The X-ray CT images show the core still attached to the face sheet with some core crushing. The PCA image was calculated from the first eigenvector. A comparison of the late time (1.5 - 8.3 seconds) PCA image to ultrasonic and X-ray CT inspection images are shown in figure 8 . There is good qualitative agreement between the thermography inspection image (early time analysis) compared to the ultrasonic and $\mathrm{X}$-ray CT image.

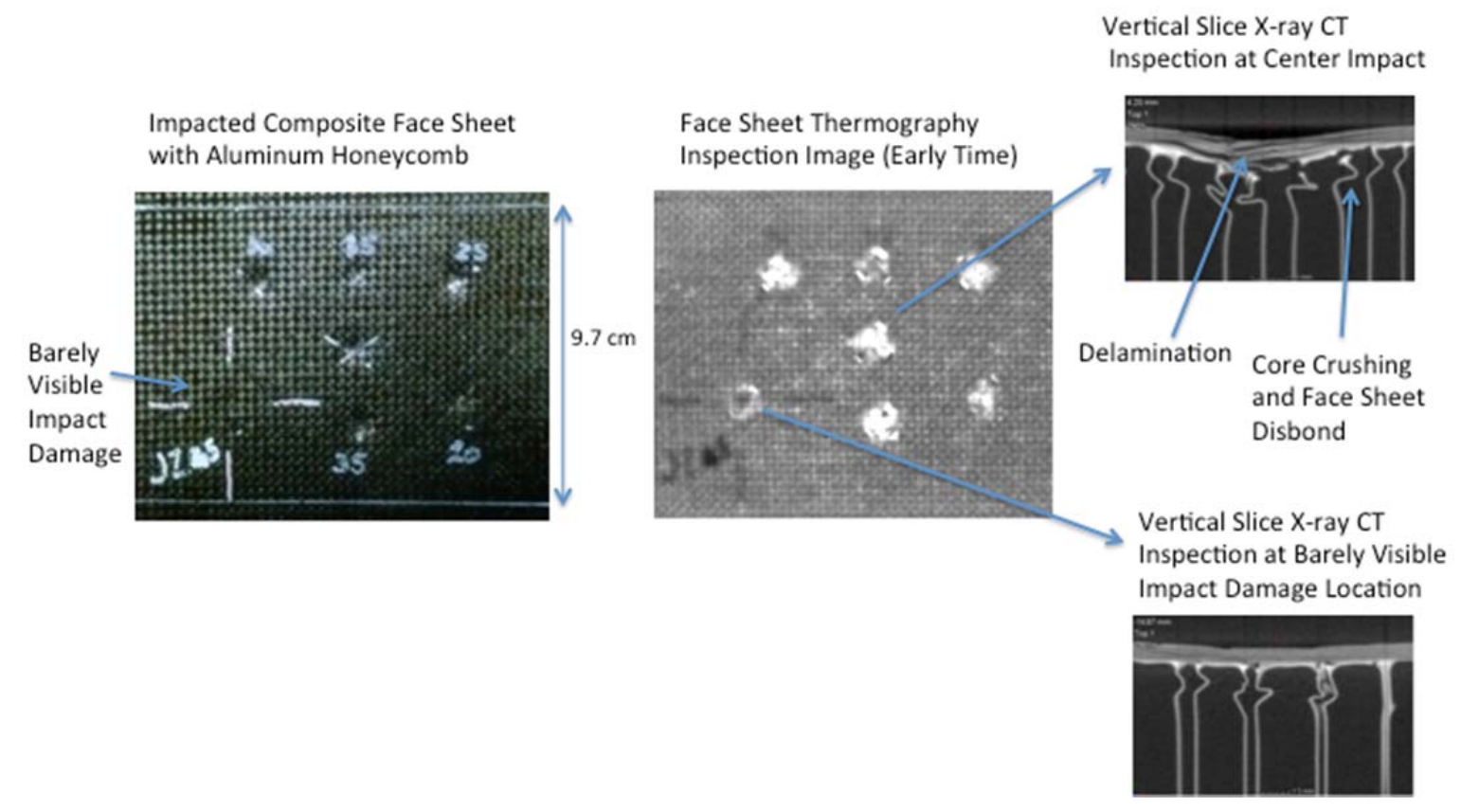

Figure 7. Thermography inspection results (early time) of an impacted sandwich structure with X-ray CT.
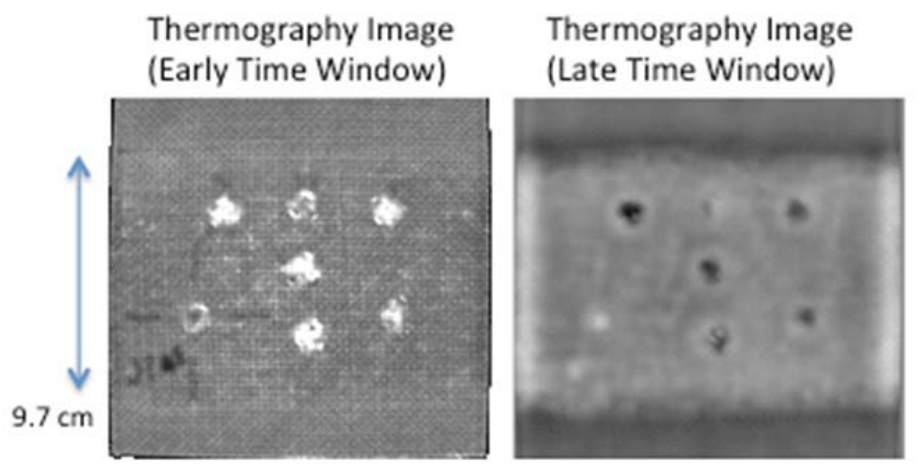

\section{$\mathrm{X}$-ray CT Image}

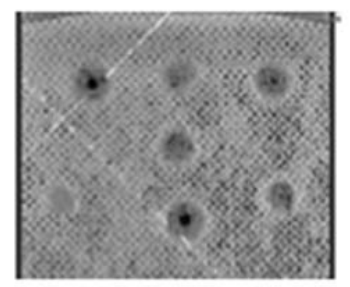

Ultrasonic Image $(10 \mathrm{MHz})$

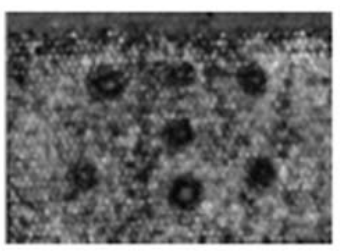

Figure 8. Thermography inspection results (late time) of an impacted sandwich structure. 
Another sample, containing a face sheet crack, impact damage and core damage (cut core) was inspected. Shown in figure 9 is an early time $(0.33-1.5$ seconds) PCA image of the honeycomb sample with a face sheet crack and impact damage defects. The bottom left image of figure 9 is the late time $(5.0-8.3$ seconds) PCA image of the honeycomb sample showing the face sheet to core disbonds along the edge. A later start time (5.0 seconds) was used in the PCA processing to image the cut core areas and this was found to provide optimal results. The later time requirement is most likely due to some portion of the aluminum core still attached to the face sheet. This can be clearly seen in the left X-ray CT images of figure 10 where some of the damaged core is still attached to the face sheet and therefore later times were required to produce the surface temperature contrast. Shown in figure 10 is a close up area of the boxed region of figure 9 where the impact damage is over an area of cut core. The early time image and late time image (shown in figure 10) clearly reveals the face sheet impact damage and the face sheet to core disbond respectively. The face sheet to core disbond region is larger than the impact damage. The cut core cannot be detected under the impact damage area as expected due to the delamination blocking the heat flow.

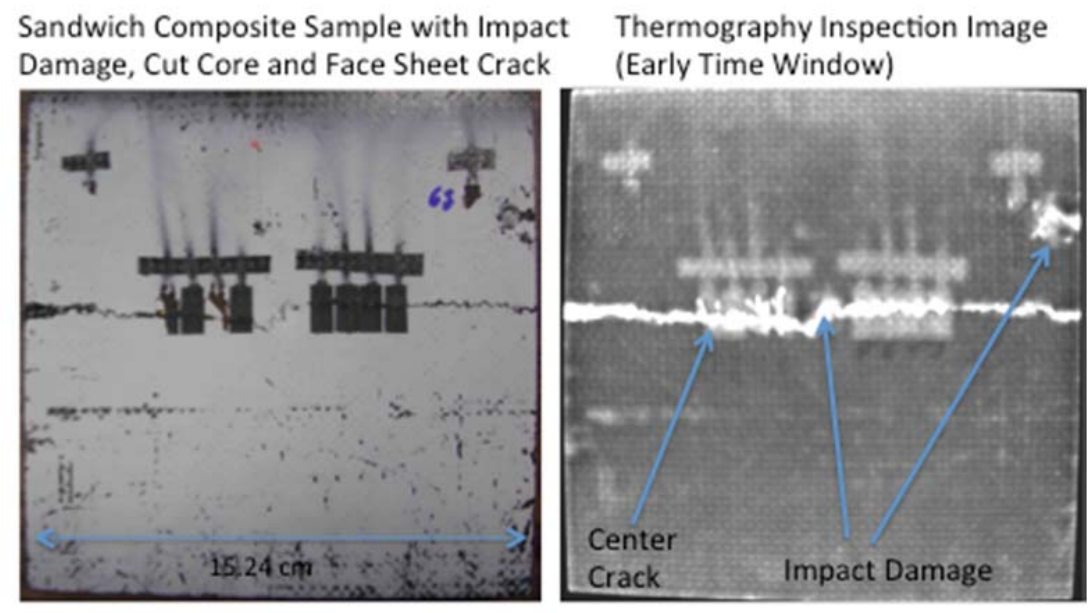

Thermography Inspection Image (Late Time Window)

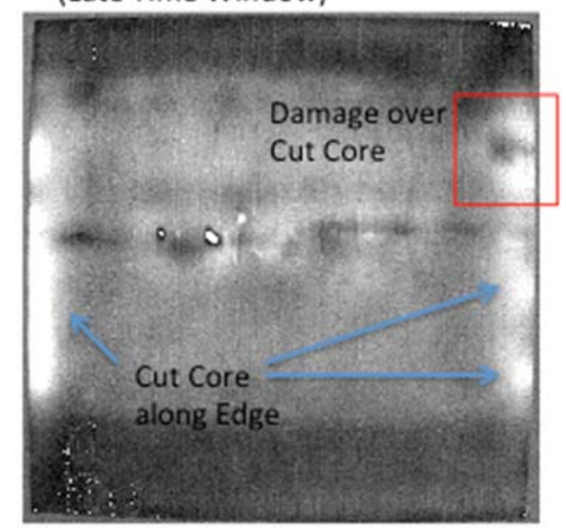

X-ray CT Image Horizontal Slice

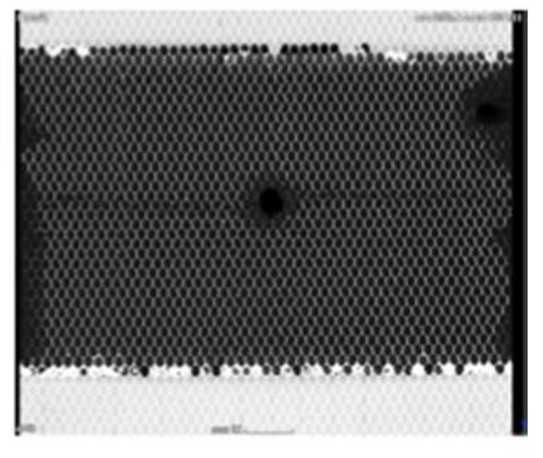

Figure 9. Thermography inspection results (early and late time) of the damaged sandwich panel with X-ray CT.

\section{SUMMARY}

A method is presented to detect damage in aluminum core composite face sheet sandwich structures using PCA and time window analysis. The early time window PCA image using the first eigenvector was applied to detect face sheet impact damage. The late time window PCA image using the second eigenvector was applied to detect the face sheet to core 
disbond areas. The FEM analysis was helpful in selecting the eigenvector number and determining the optimal time windows. These results were confirmed with X-ray $\mathrm{CT}$ and ultrasonic measurements.

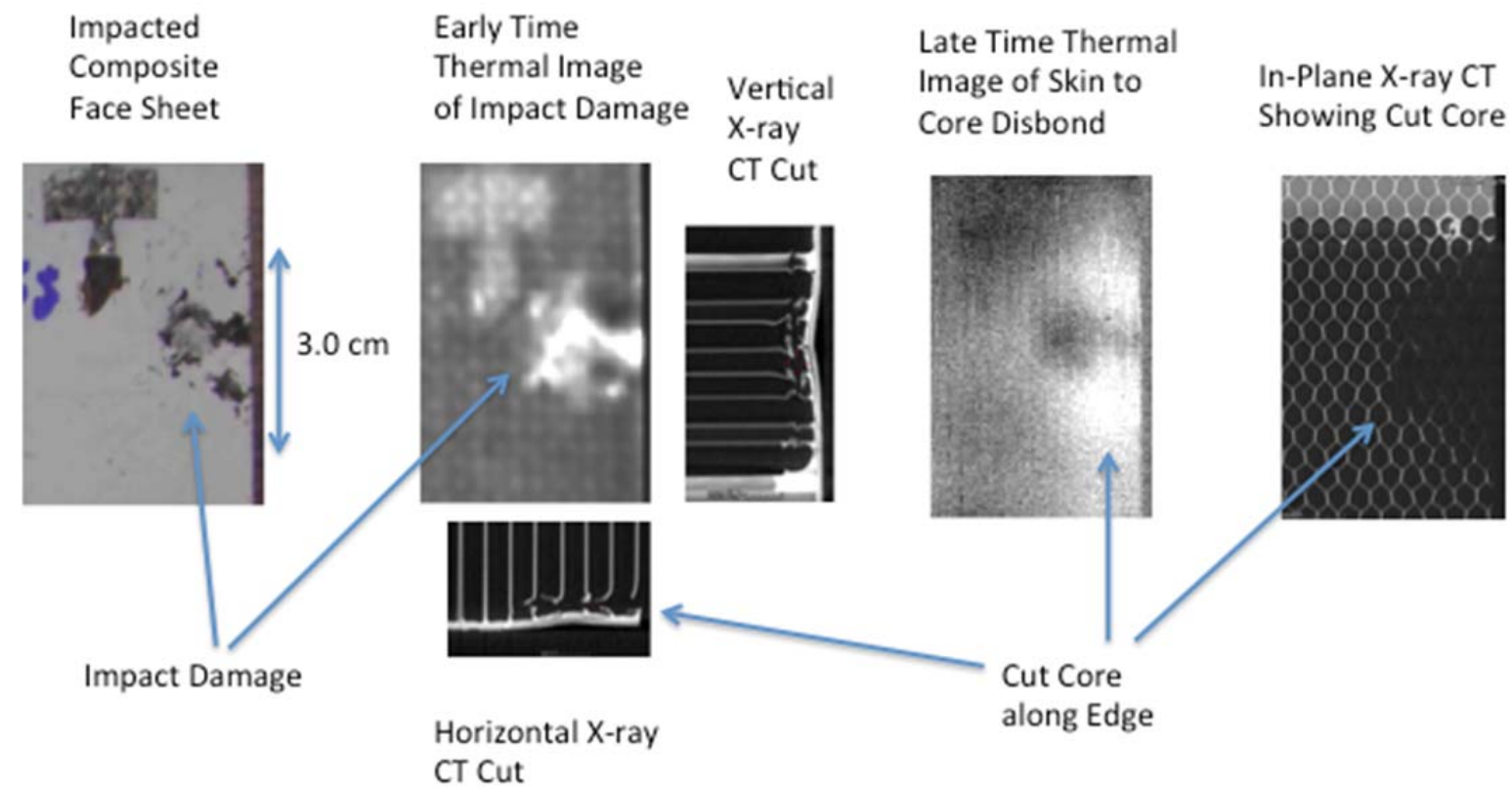

Figure 10. Thermography inspection close up of impact damage over cut core with X-ray CT.

\section{REFERENCES}

[1] S Sfarra et al, "A comparative investigation for the nondestructive testing of honeycomb structures by holographic interferometry and infrared thermography“", 2010 J. Phys.: Conf. Ser. 214012071.

[2] Clemente Ibarra-Castrando et al, "Comparative Study of Active Thermography Techniques for the Nondestructive Evaluation of Honeycomb Structures", Research in Nondestructive Evaluation Volume 20, Issue 1, 2009.

[3] M. Genest, M. Brothers, C. A. Beltempo, R. S. Rutledge, "Nondestructive Evaluation of Aluminum Honeycomb Sandwich Structures", NATO Unclassified, STO-MP-AVT-224, 2013.

[4] T. Southward, D.P. W. Horrigan, G. D. Mallinson, and K. Jayaraman, "Failure of Sandwich Composite Structure Containing Face-sheet/Core Disbonds - An Experimental Study", $5^{\text {th }}$ Australasian Congress on Applied Mechanics, ACAM 2007 10-12 December 2007, Brisbane, Australia.

[5] Carslaw H. S. and Yeager J. C., Conduction of Heat in Solids, 2nd. Ed. Oxford Univ. Press, 1959.

[6] A. Talbot, IMA J Appl Math 23 (1): 97-120, (1979).

[7] S. M. Shepherd, J. M. Lhota, and T. Ahmed, "Measurement Limits of Flash Thermography", Proceedings of SPIE, Thermosense XXXI, edited by Douglas D. Burleigh and Ralph B. Dinwiddie, Vol. 7299, 2009.

[8] N. P. Devadas, G. Sunilkumar, and R. Sajeeb,"Finite Element Analysis of Flat Joints in Metallic Honeycomb Sandwich Beams", Journal of Mechanical and Civil Engineering, ISSN: 2278-1684, Vol. 3, Issue 2, Sept-Oct. 2011.

[9] LISA Finite Element Analysis, software version 8.0.0, 2013.

[10] J. N. Zalameda, "Heat Source Finite Pulse Time Effects in Thermal Nondestructive Evaluation," Materials Evaluation Journal, American Society for Nondestructive Testing, Vol. 60, Nos.3, March, 2002, pp.425-429.

[11] N. Rajic, "Principal Component Thermography for Flaw Contrast Enhancement and Flaw Depth Characterisation in Composite Structures", Composite Structures, Vol. 58, pp. 521--528, 2002.

[12] K. E. Cramer and W. P. Winfree, "Fixed Eigenvector Analysis of Thermographic NDE Data", Proceedings of SPIE, Thermosense XXXIII, edited by Morteza Safai and Jeff Brown, Vol. 8013, 2011. 
Filename: 1f99-18758_final-2014_spie_thermosense_jnz_vfinalv2

Directory: $\quad$ C: $\quad$ Users $\backslash w b a i z e \backslash A p p D a t a \mid L o c a l \backslash T e m p \backslash 2$

Template:

C: $\backslash$ Users $\backslash$ wbaize $\backslash A p p D a t a \backslash R o a m i n g \backslash$ Microsoft $\backslash T e m p l a t e s \backslash$ Normal.

$\operatorname{dotm}$

Title:

Subject:

Author: mattn

Keywords:

Comments:

Creation Date: $\quad$ 4/21/2014 10:45:00 AM

Change Number: $\quad 20$

Last Saved On: $\quad$ 4/21/2014 1:08:00 PM

Last Saved By: Joseph Zalameda

Total Editing Time: 102 Minutes

Last Printed On: $\quad$ 5/15/2014 7:56:00 AM

As of Last Complete Printing

Number of Pages: 8

Number of Words: $\quad 3,045$ (approx.)

Number of Characters: 17,362 (approx.) 